Jan Gaebel*, Carsten Bockelmann, Robert Wendlandt, Armin Dekorsy, Juliane Neumann, Torsten Musiol, Thomas Neumuth, Max Rockstroh

\title{
Requirements for 5G Integrated Data Transfer in German Prehospital Emergency Care
}

\begin{abstract}
Data from preclinical emergency care is often not available in an integrated, electronic way. Data flow between ambulances and trauma centers lacks a communication approach that allows for efficient aggregation, transmission and reuse. We present the results of the requirement analysis for 5G-supported emergency care scenarios. With the illustarted requirements and derived structural consequences, we conclude with a proposal that will allow us to provide mechanisms and technologies to enable integrated emergency communication for preclinical care using modern communication technologies.
\end{abstract}

Keywords: Emergency Medical Service, Trauma Team, Emergency Room, Device Connectedness, Integrated Data Access, Mobile Data, 5G.

https://doi.org/10.1515/cdbme-2020-3003

\section{Introduction}

Health information technology is being established increasingly throughout all areas of healthcare. This progress not only enables a comprehensive documentation along the clinical pathways but also ensures the crucial continuity of care. However, emergency medical services (EMS) often lack the integration into hospital IT infrastructure necessary to request and send patient data [1]. In general, medical data gathered during pre-clinical emergency care cannot be efficiently managed or communicated with other healthcare providers [2]. Relevant patient data needed to assess the current situation, i.e. patient history, allergy status or

\footnotetext{
${ }^{*}$ Corresponding author: Jan Gaebel: University Leipzig, Faculty of Medicine, ICCAS, Semmelweisstr. 14, Leipzig, Germany, e-mail: jan.gaebel@medizin.uni-leipzig.de Carsten Bockelmann, Armin Dekorsy: University of Bremen, Dept. of Communications Engineering, Bremen, Germany Robert Wendlandt: University Medical Centre SchleswigHolstein, Clinic for Orthopaedic and Trauma Surgery, Laboratory for Biomechanics, Lübeck, Germany

Torsten Musiol: MECSware $\mathrm{GmbH}$, Ratingen, Germany Juliane Neumann, Thomas Neumuth, Max Rockstroh: University Leipzig, Faculty of Medicine, ICCAS, Leipzig, Germany
}

medication plan, are usually only available in paper form, e.g. at the patient's home environment.

In Germany, pre-hospital emergency services rely on ground-based ambulances and airborne rescue helicopters. Both are staffed with paramedics and emergency physicians [3]. Research projects and commercial systems established in various regions of Germany have been able to support emergency services through provisioning of improved communication and documentation.

Telemedical pre-notification: Paper-based documentation is frequently used in emergency services. From a system for tablet-based documentation, Eder et al. developed a system, which connects ambulances and clinics [4]. Medical data gathered in the ambulance is transmitted alongside with position data and an expected arrival time into the hospital. This data is used to prepare the handover of the emergency patient to the hospital as well as to alarm the required medical specialists while the ambulance is still on its way.

Telemedical consultation: To counteract shortcomings of separately dispatched emergency physicians in Germany, telemedical consultation for on-site paramedics by stationary emergency physicians was established by the TeleMedic [Telenotarzt] project initially for the City of Aachen. It is in use in different regions throughout Germany. Speech, images, videos and medical data such as a live ECG are transmitted in real-time via mobile phone networks between on-site paramedics and a teleconsultation center.

The examples given above for two different use cases of telemedical services in EMS are based on proprietary communication protocols and commercial devices. Consequently, the systems only gained regional distribution. Nonetheless, they are valuable tools for improving emergency medical services [5].

The MOMENTUM research project, funded by the Federal Ministry of Education and Research, aims to develop a standardized interconnection of medical devices used in preclinical emergency services as well as a completely integrated communication approach between all medical entities involved in preclinical emergency care. In pursuing this project, we are trying to overcome the limitations of the systems that have been implemented to date, each of which only realizes parts of in integrated communication from 
ambulance via emergency command center into the hospital and vice versa. In this paper, we illustrate the results of the requirement analysis.

\section{Methods}

\subsection{G specification and medical use cases}

The term " $5 \mathrm{G}$ " is an unspecific marketing term that is used with very different meanings and in different contexts. When talking about the mobile communications system 5G, the standardization organization 3rd Generation Partnership Project (3GPP) defines releases that make up the cellular communication systems used today (i.e. from GSM to LTE). With release 15 (Rel. 15) 3GPP introduced the first "5G"System with New Radio (NR) as its novel air interface (i.e. layers 1 and 2 ) and the new $5 \mathrm{G}$ core $(5 \mathrm{GC})$ that handles higher layer functions like authentication, mobility or the gateway to public networks. Within 3GPP 5G standardization continues with parallel tracks on the finalization of Rel. 16 (expected Q3/20) and ongoing work on Rel. 17 (expected to finish 09/21).

Medical service requirements are discussed in 3GPP since late 2018 initially as part of Release 16 and now as an ongoing study item in the Systems Architecture (SA) working group for Release 17. The current status is documented in the technical report TR 22.826 [1], which lists several different medical applications and discusses functional as well as performance requirements that are relevant for cellular communication systems like 5G. Analyzed medical applications range from simple video duplication or robot-aided surgery to very challenging remote surgery. The use cases most relevant for our purposes are:

- Mobile specialist practice

- $\quad$ Patient monitoring inside ambulances

- Cardiac telemetry outside the hospital

Each use case is characterized by functional requirements, usually concerning security, privacy and service reliability. The performance requirements are typically detailed on the application level like video and audio streams for communication and diagnostics, DICOM data like MRI/CT scans, haptic feedback data, telemetry and so forth. In the following we want to highlight and summarize the 3GPP requirements.

Interesting functional requirements:
Reservation service requirements for medical services for guaranteed performance

- Clock synchronization service level requirements related to global clock domain management

Summarized performance requirements:

- Ambulance setting limited to $4 \mathrm{~K}$ compressed audio/video, vital signs and a $2 \mathrm{~K}$ low fps data stream for diagnostic video (CT scan)

- Mobile specialist practice includes very high data rate demands with $4 \mathrm{~K}$ stereo video, fast MRT/CT scan data transmission and multiple additional streams for diagnostic video

Current 3GPP use cases detail rather generic requirements on data rate, latency and availability, but lack detailed analysis of current medical technologies, their capabilities and restrictions. Section 3.1 provides examples of specific requirements for typical medical data in German ambulances that are not covered by this view.

\subsection{Technical prerequisites}

The German Society of Anesthesiology and Intensive Care Medicine responded to the technological progress and recent growing demands on support with a proposal of minimum technical requirements for telemedicine in prehospital emergency care [6]:

- Availability of bidirectional audio transmission, unidirectional video transmission

- Continuous transmission of vital sign/monitoring data, low clinically irrelevant latency

- Secure data transfer, availability in $95 \%$ of the operation, state of the art data encryption

- Redundancy in communication infrastructure as a fallback

- Data management compliant to GDPR, medical data privacy laws etc.

- Availability of GPS data of ambulance and relevant logistical data, support of operation management and choice of target clinic

- Digital medical documentation for ambulance personnel, forensically sound and compliant to minimum emergency dataset MIND3 [7]

This proposal is based on for German EMS available communication technologies, UMTS, LTE and TETRA radio (Terrestrial Trunked Radio). The previously mentioned systems are using these technologies to provide their subset of implemented functionalities of [6]. 


\section{Results}

After observations and investigations in both German emergency medical services and trauma centers, we propose to extend the requirements regarding the following use cases:

- Interconnectedness of medical devices and other technical equipment inside the ambulance

- Integration/connection to "Telematikinfrastruktur" ${ }^{1}$ with identification of patient and check for health data

- Distributed processing/analysis of collected medical data inside whole infrastructure

- Possibility of the integration of additional devices/technologies (e.g. mobile ultrasound or other imaging or sensor technology)

- Comprehensive electronic patient record with continuous monitoring documentation and automated data entry (where/when needed)

- Alerting of the trauma team in the target clinic with detailed information and individual call-in of medical specialists

\subsection{Data \& devices in ambulance vehicle}

German ambulances are equipped according to European standard DIN EN 1789 [6]. Due to German federalism, each German federal state can define the features in detail and may also require additional equipment. Regarding medical devices or technical equipment in general, ambulances contain an ECG monitor with defibrillator, a respirator, syringe driver, suction pumps, TETRA radio and GPS navigation device. Additionally, mobile computers are sometimes available for electronic documentation.

To fulfil the aforementioned requirements, we considered additional devices, e.g. mounted or mobile camera systems or medical devices with Service-oriented Device Connectivity [9]. To analyze and assess the technical environment needed, we estimated the potential traffic and data amount using exemplary data from vendor information/websites or scientific publications.

Our research resulted in very a heterogenous set of characteristics for different medical applications. Apart from existing technologies, e.g. mobile video transmission, and innovative approaches like augmented reality applications,

1 German health care system telematics infrastructure to access a patient's core data or relevant health data standard emergency care actions present a challenge to communication technologies. For example, monitoring of an emergency patient comprises of different parameters, that when transferred to other a remote party (e.g. leader of the trauma center in the target clinic), require different transmission strategies. The following list contains only a few examples of emergency medical procedures and their properties for successful transfer:

- ECG monitoring \& oxygen saturation: continuous stream, low latency, minimal packet loss tolerable

- Blood pressure: repeated measurement, low latency, lossless transmission

- Respiratory monitoring: continuous stream, low latency, minimal packet loss tolerable

- Telemedical adjustment of respiration parameters: minimal latency, lossless transmission

These examples illustrate the heterogenous nature of different medical measurements or activities. Regarding the 3GPP release, these activities cannot be covered by the given use case descriptions. Further conceptualization is required to integrate the entire medical process with the desired benefits.

\subsection{G-Scenario architecture}

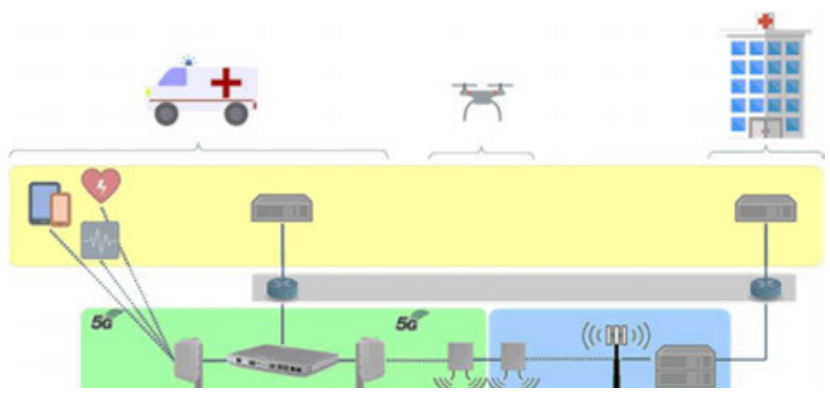

Figure 1: Proposed architecture for emergency services. Top most involved communication partners are shown. An ambulance connected to a hospital uses a drone to boost its connection to public infrastructure. Middle layer shows the application view with medical applications and servers and bottom layer describes the involved $5 \mathrm{G}$ system with a small cell inside the ambulance to connect medical devices and (potentially mobile) gateway to public networks.

To connect an ambulance (top left) to the hospital (top right) for reliable two-way communication, we propose an architecture as shown in Figure 1. The ambulance uses a drone (top middle) that can be flexibly placed to enhance connection to public services. This is of special importance in rural areas to enhance signal quality, e.g., in mountain terrain or within a valley, but can also be useful in urban areas where densely placed buildings are blocking radio reception. 
On the application level (middle layer) the ambulance will run its own medical network with a variety of medical equipment connected to an appropriate server that coordinates data forwarding and local processing. This server is the endpoint towards the hospital medical network and applications.

The $5 \mathrm{G}$ network (bottom layer) is structured to support reliable connections within the ambulance as well as with a public network. Thus, the ambulance provides its own private cellular network with a $5 \mathrm{G}$ small cell handling traffic of all wireless medical equipment within the ambulance. Note, that this requires appropriate bandwidth for a mobile private network which is not covered in current regulatory rules. Furthermore, the private ambulance small cell must provide coverage outside the ambulance to enable mobile use of all equipment in the vicinity. Access should be available to other devices/vehicles in a rendezvous system, e.g. by simple NFCbased on-site authentication services.

The drone serves as a gateway to the public network and can be either stowed on the ambulance or in the air with the goal to optimize reception. Currently, to realize the gateway functionality we propose the use of two modems, one connected to the public network and one to the private small cell network served by the ambulance. In future $5 \mathrm{G}$ solutions, this might be integrated into a single device. Alternatively, a tether could be used to connect the drone to the ambulance network also solving potential power issues, but constraining movement.

\section{Discussion \& conclusion}

In this paper, we present the requirements for an integrated communication system and device connectivity in preclinical emergency care.

Modern telecommunication technologies enable the development of sophisticated assistance systems. However, to ensure the establishment in medical practice and the acceptance of the potential users, systems must meet the prevalent requirements. We analyzed existing conditions in German preclinical emergency care and related them to potential solutions. German federalism complicates the definition of generally valid solutions. But progress and modern technologies from other communities, can help achieve the goal. With the introduction of Rel. 15, the 3GPP presents specifications that enable authentication, mobility or the gateway to public networks. Given the necessary security measures, this standard may allow preclinical entities to use mobile communication channels to transfer their data to all stakeholders. Technical standards from clinical/surgical settings, e.g. IEEE 11073 SDC [9], enables the connectivity of medical devices inside the ambulance and even beyond the boundaries of the ambulance vehicle. This, on the other hand, allows for an integrated health record of the patient that can be made available to every attending paramedic or physician.

The MOMENTUM consortium will continue to conceptualize and implement technologies with integrated communication systems for preclinical emergency care.

\section{Author Statement}

This work is funded by the German Federal Ministry of Education and Research (BMBF) with grant number 16KIS1030 - MOMENTUM - Mobile Medizintechnik für die Notfallmedizin. The statements made herein are solely the responsibility of the authors.

The authors state that they have no conflict of interests.

\section{References}

[1] Martin TJ, Ranney ML, Dorroh J, Asselin N, Sarkar IN. Health Information Exchange in Emergency Medical Services. Appl Clin Inform. 2018;9(4):884-91.

[2] Landman AB, Rokos IC, Burns K, Van Gelder CM, Fisher RM, Dunford JV, u. a. An open, interoperable, and scalable prehospital information technology network architecture. Prehosp Emerg Care. Juni 2011;15(2):149-57.

[3] Brokmann JC, Felzen M, Beckers SK, Czaplik M, Hirsch F, Bergrath S, u. a. Telemedizin: Potenziale in der Notfallmedizin. Anästhesiol Intensivmed Notfallmed Schmerzther. Februar 2017;52(2):107-17.

[4] Eder PA, Dormann H, Krämer RM, Lödel SK, Shammas L, Rashid A (2018) Telemedizinische Voranmeldung durch den Rettungsdienst bei Schwerverletzten. Notfall Rettungsmed (2019) 22: 37

[5] Eder PA, Reime B, Wurmb T, Kippnich U, Shammas L, Rashid A. Prehospital telemedical emergency management of severely injured trauma patients. Methods of information in medicine. Georg Thieme Verlag KG; 2018;57(05/06):23142.

[6] Präsidium der DGAI. Telemedizin in der prähospitalen Notfallmedizin, Strukturempfehlung der DGAI. Anästh Intensivmed 2016; 57: 160-166

[7] Messelken M, Schlechtriemen T, Arntz H-R, Bohn A, Bradschetl G, Brammen D, u. a. Minimaler Notfalldatensatz MIND3. Notfall Rettungsmed. 1. Dezember 2011;14(8):64754.

[8] DIN EN 1789:2014-12: Medical vehicles and their equipment - Road ambulances; German version EN 1789:2007+A2:2014; https://dx.doi.org/10.31030/2240534

[9] Kasparick M, Schmitz M, Andersen B, et al. (2018). OR.NET: a service-oriented architecture for safe and dynamic medical device interoperability. Biomedical Engineering / Biomedizinische Technik, 63(1), pp. 11-30. Retrieved 23 Mar. 2020, from doi:10.1515/bmt-2017-0 